\title{
An auto-balancer device for high spin-drying frequencies (LoWash Project)
}

\author{
Christian Clerc ${ }^{\mathrm{a}}$, Alexandre Carbonelli and Romain Augez \\ VIBRATEC, 28 chemin du Petit Bois, 69131 Ecully Cedex, France
}

\begin{abstract}
Auto-balancing or active control balancing can be efficient solutions for high speed rotors with changing out-ofbalance loads like washing machines in spin-drying mode. In the LoWash EU project, Vibratec is in charge to design, to build and to validate a balancing system for reducing the vibrations at high spin-drying speeds. The system is based on two trolleys rolling in a ring linked to the drum. The trolley shape allows a ring cross section optimization and they are equipped with a mechanism for escaping the disadvantage encountered at low speeds by similar devices. Analytical and multi-body models are first made for understanding the mechanisms, highlighting the driving parameters and drawing the final design of a first prototype which is inserted in a washing machine drum. Different tests are carried out for different initial unbalances and different rotation speeds: the residual unbalance is measured by means of a set of accelerometers mounted on the tub, while the mobile masses behaviour is observed by means of a large aperture swift camera. The test results highlight the auto-balancer high efficiency but also the sensitivity to geometrical defects which should be corrected in the next systems. According the theory, the balancing is efficient when the rotation frequency is significantly greater than the hanging frequencies. The multi-body model relevance is also demonstrated. A washer-dryer prototype including an auto-balancer second prototype and two other innovations, regarding thermal exchange efficiency and drum insulation, will be tested in operating conditions.
\end{abstract}

\section{Introduction}

The out-of-balance loads are the main source of vibration for the rotating machines. It is especially the case of the washing machines which are submitted to unbalances which are not only high but also changing, in magnitude and location, during the spin-drying cycle. In this case, an active control balancing or an auto-balancer are required. This paper deals with the second solution which has been designed and assessed within the LoWash EU project which aims at developing a new innovative washer-dryer. After few considerations about the assumptions and the theory of rotor balancing, the second section presents the simulations models which have been used for designing a first prototype of an auto-balancer device.

The third section is dedicated to the measurements and the results analysis led on the first prototype. The results allow investigating the balancing device behaviour and assessing the global efficiency. The multi-body model results are also assessed by comparison to the measurement results.

The fourth section presents the results obtained on a second prototype, aimed to be inserted in the final washer-dryer prototype, and in which some improvements, deduced from the first prototype results are implemented.

The conclusion is made in the fifth section and dedicated to the balancing device efficiency, its potential improvements and its potential extensions to other industrial applications.

\footnotetext{
${ }^{a}$ Corresponding author: christian.clerc@vibratec.fr
}

\section{Theory and simulation}

\subsection{Theory}

Many studies ([1] to [6]) deal with the automatic balancing by mobile masses (in general balls). The most important remark concerns the rotational speed of the drum $\omega$, which has to be higher than the last suspension natural frequency $\omega_{n}$ in order to have the correcting masses out of phase with the unbalance ([1], see Fig. 1).

Let us consider the hanged system ([2]) on the Fig. 2 constituted by a rotor (mass $\mathrm{M}$ ), an unbalance (mass $\mathrm{m}_{\mathrm{u}}$ ) and several balancing masses $m_{i}$.

The equations of motion are derived from Lagrange's equations which lead to:

$$
\begin{aligned}
& M^{\prime} \ddot{x}+c_{x} \dot{x}+k_{x} x=m_{u} e \omega^{2} \sin (\omega t) \\
& \quad-\sum_{i=1}^{i=n} m_{i} l_{i}\left(\ddot{\beta}_{1} \sin \left(\beta_{i}+\omega t\right)-\left(\dot{\beta}_{1}+\omega\right)^{2} \cos \left(\beta_{i}+\omega t\right)\right) \\
& M^{\prime} \ddot{y}+c_{y} \dot{y}+k_{y} y=m_{u} e \omega^{2} \cos (\omega t) \\
& \quad+\sum_{i=1}^{i=n} m_{i} l_{i}\left(\ddot{\beta}_{1} \sin \left(\beta_{i}+\omega t\right)+\left(\dot{\beta}_{i}+\omega\right)^{2} \cos \left(\beta_{i}+\omega t\right)\right)
\end{aligned}
$$

$$
\begin{aligned}
& m_{i} l_{i} \ddot{\beta}_{i}+\delta_{i} l_{i} \dot{\beta}_{1}=-m_{i}\left(\ddot{x} \cos \left(\beta_{i}+\omega t\right)\right. \\
& \left.-\ddot{y} \sin \left(\beta_{i}+\omega t\right)\right), \quad i=1 \ldots n .
\end{aligned}
$$

Where,

$M^{\prime}$ is the total mass of the system, thus $M^{\prime}=M+m_{u}+\Sigma_{i=1}^{n} m_{i}$

This is an Open Access article distributed under the terms of the Creative Commons Attribution License 2.0, which permits unrestricted use, distribution, and reproduction in any medium, provided the original work is properly cited. 


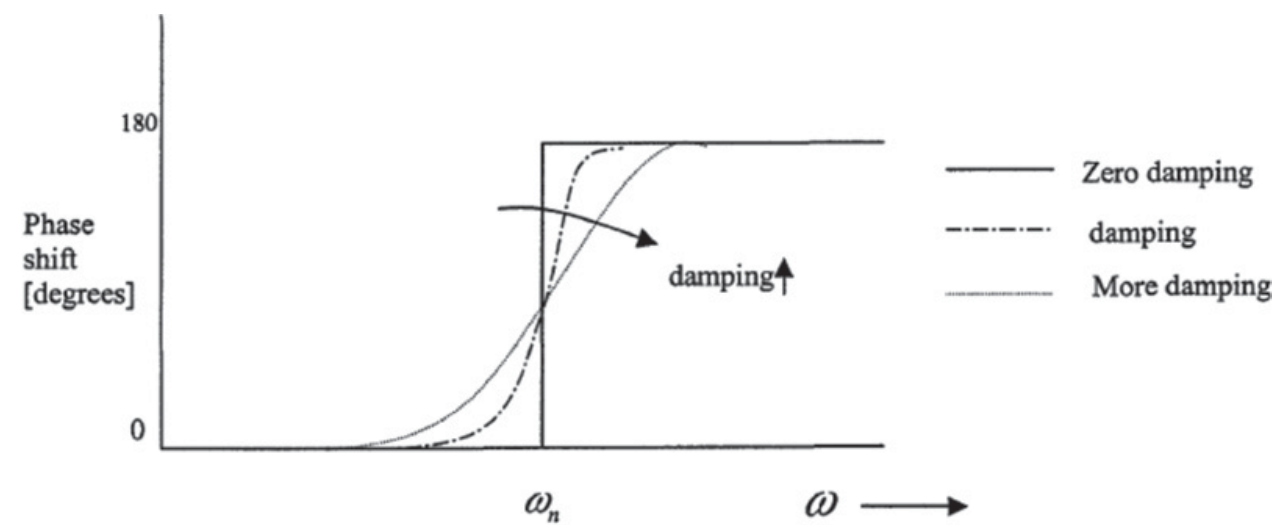

Figure 1. Phase shift between the correcting masses COG and the unbalance [1].

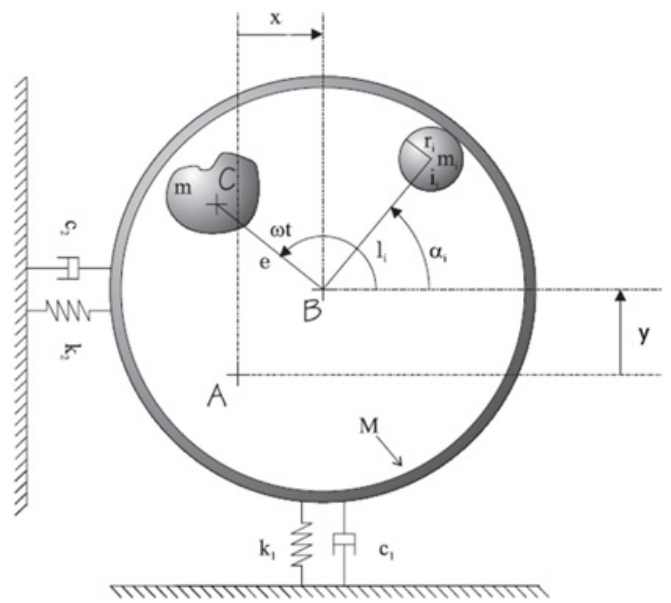

Figure 2. The auto balancing model from [2].

$c_{j}$ is the suspension damping,

$k_{j}$ is the suspension stiffness,

$e$ is unbalance radius,

$l_{i}$ is the radius at which the correcting masses are located,

$\delta_{i}$ is the damping acting on the mass $i$,

$\beta_{i}$ is the angle between the unbalance and the correcting mass $i$.

\subsection{Multi-body model}

As a multi-body is planned for designing the auto-balancer, a model of the auto-balancer included in a single plane rotor is validated by comparison to the analytical model (see Fig. 3) issued from the theory and implemented in Matlab software.

\subsection{Parameters analysis and final design}

By considering the previous results, a final Adams model (Fig. 3) can be proposed which consists in inserting a ring model in the global washing machine model and replacing the balls by shaped trolleys.

This replacement aims at optimizing the ring section while minimizing the mobile masses number and cancelling the disadvantage of such a system at low speed
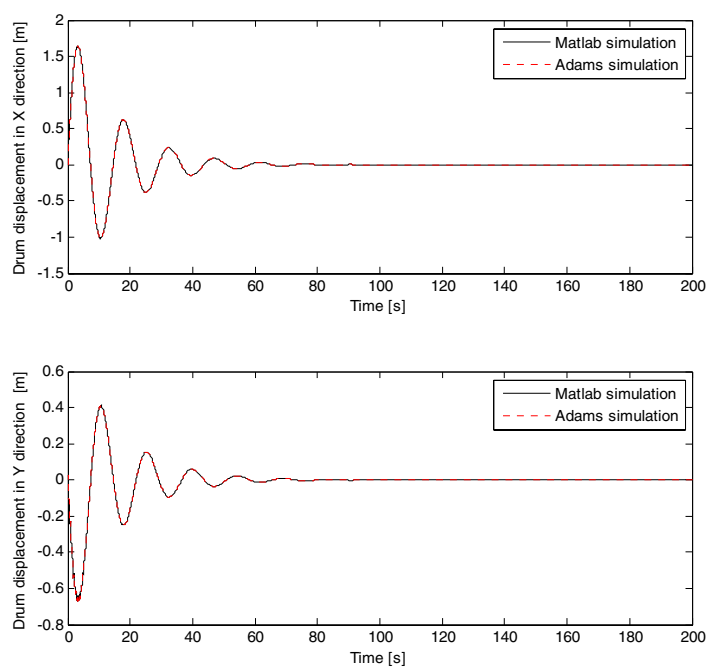

Figure 3. Drum displacements with 2 correcting masses. Analytical model vs. Adams software.

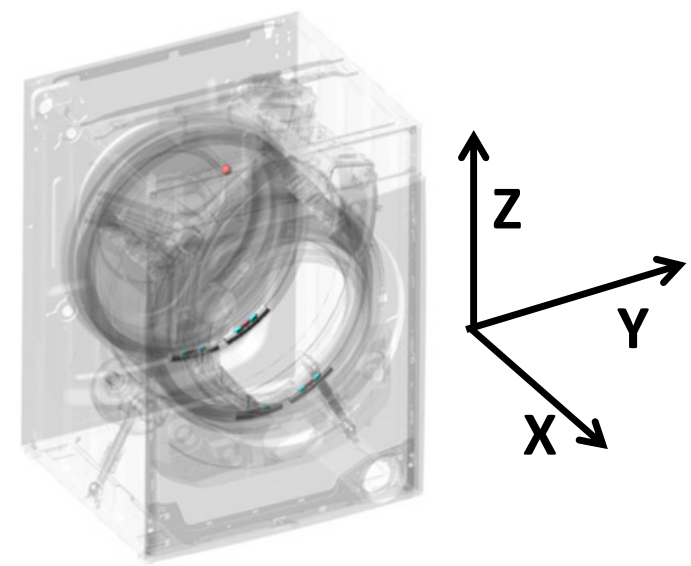

Figure 4. Complete model with 2 balancing rings and Cartesian axes system.

(acoustic and vibration) by means of a mechanism able to block the mobile masses.

Different parametric calculations are led with the model in order to investigate the performance sensitivity 


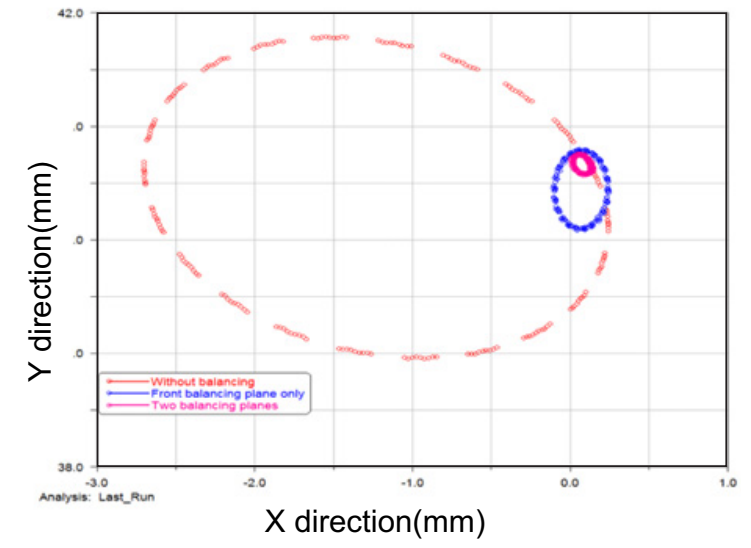

Figure 5. Drum displacements. Comparison between 1 and 2 balancing planes.

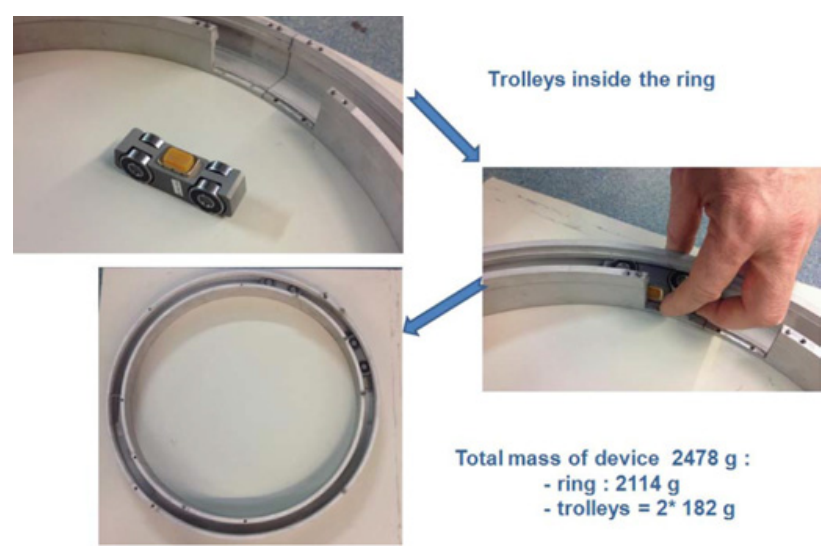

Figure 6. $1^{\text {st }}$ prototype. Rolling ring and trolleys.

to geometric defects and to determine the conditions to fulfil for an efficient balancing. The most important is the comparison of the efficiency between 1 balancing plane and 2 balancing planes (see Fig. 5) which shows that the benefits of a second plane is low (regarding the industrial constraints) and that one balancing ring is sufficient (the drum is a short rotor with a length lesser than the diameter).

\section{Measurements $-1^{\text {st }}$ prototype}

\subsection{Experimental set-up and test matrix}

A first prototype is built (see on Fig. 6), based on the simulation results. It is fixed inside a washer dryer drum for tests. The aim is to validate the auto-balancer and to assess its efficiency.

The experimental set-up includes:

- A laser speed sensor for the rotation speed measurement.

- 4 3-D accelerometer for the tub and drum displacements measurements (the main used result is the $\mathrm{YZ}$ plane trajectory built from the average magnitude in $\mathrm{Y}$ and $\mathrm{Z}$ direction).

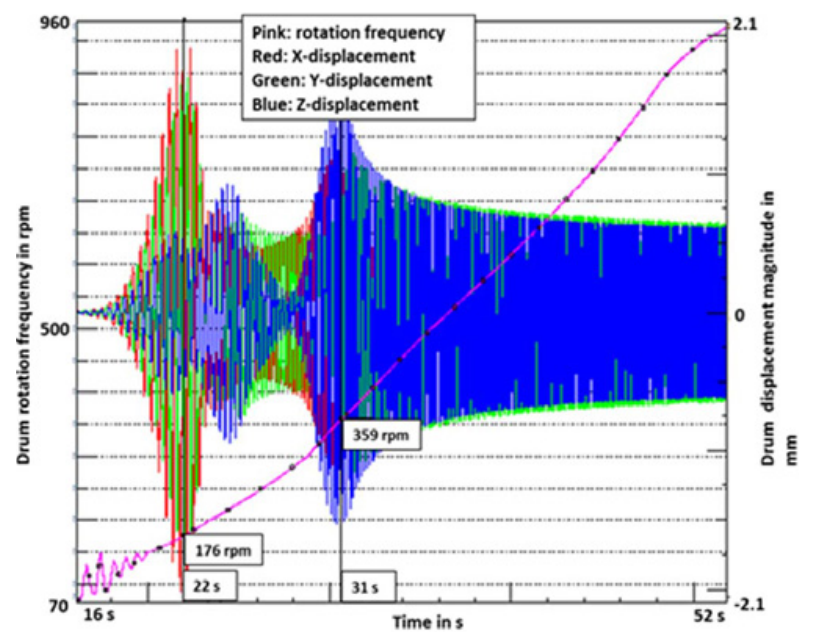

Figure 7. $1^{\text {st }}$ prototype. Drum displacement during a drum speed up.

- A wide aperture camera the frequency of which can be synchronised or slightly unsynchronised with the drum rotation.

The rotation speed is manually driven by a specific power supply.

\subsection{Preliminary tests}

The system is supposed to be efficient after the first natural frequencies (the hanging modes frequencies). The hanging mass is made of the tub and the equipments fixed on it (including the drum). From one side, a short experimental modal analysis is carried out in order to identify the natural frequencies, from the other side the resonances are identified during a drum speed up (see Fig. 7).

The resonances are located from $180 \mathrm{rpm}(3 \mathrm{~Hz})$ to $360 \mathrm{rpm}(6 \mathrm{~Hz})$ while the EMA frequencies are between $3.4 \mathrm{~Hz}$ to $6.5 \mathrm{~Hz}$. Operational and natural mode shapes are similar and the frequency shift $(10 \%)$ is probably due to non linearity. Thus the auto-balancer is expected to be efficient after $400 \mathrm{rpm}$.

\subsection{Running tests and analysis}

The tests are performed for different spin-drying frequencies from $600 \mathrm{rpm}$ to $1400 \mathrm{rpm}$ and 3 unbalance values: $150 \mathrm{~g}, 300 \mathrm{~g}$ and $400 \mathrm{~g}$.

The Fig. 7 presents the results obtained at different speeds with a $300 \mathrm{~g}$ initial unbalance. The dynamic equation says that the ellipse radius is proportional to the unbalance load: the external circle on the Fig. 7 shows that a $3.5 \mathrm{~mm}$ radius corresponds to a $300 \mathrm{~g}$ unbalance at $1100 \mathrm{rpm}$. The displacement magnitude is expected to become independent from the speed when it is high enough because the inertia term became significantly preponderant at high speeds. Actually, if a significant difference can be observed between $600 \mathrm{rpm}$ (which is close to the highest hanging frequency) and $1100 \mathrm{rpm}$, after $1100 \mathrm{rpm}$ the ellipse radius is constant and the results can be easily extrapolated to the higher speeds. Moreover, the Fig. 8 highlight the efficiency of the auto-balancer with a final unbalance weight located around $80 \mathrm{~g}$. 


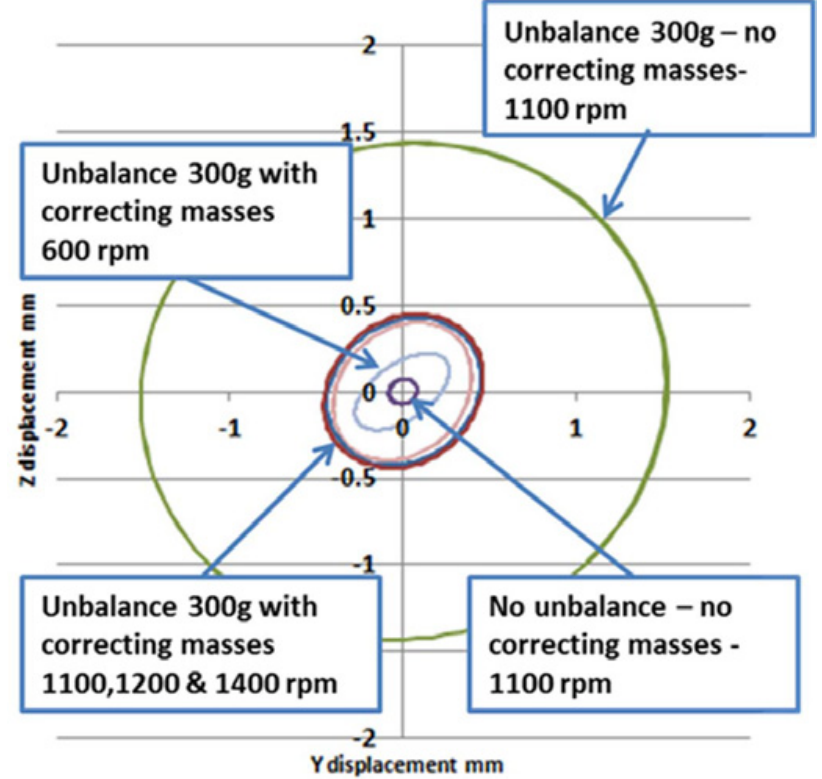

Figure 8. Drum trajectories vs. rotation speed.

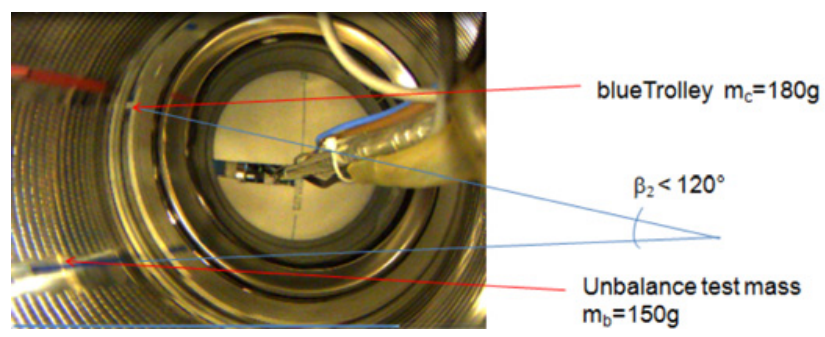

Figure 9. $1^{\text {st }}$ prototype. Trolley location at $620 \mathrm{rpm}$.

The camera is used for observing the trolleys behaviour. Colour marks are made on the drum and the trolleys for the angles estimation because of a large optical distortion (the camera cannot be centred in the drum). Video films allow observing the trolleys displacement during the time. A typical result is shown on Fig. 9 which presents a run at $620 \mathrm{rpm}$ with a $150 \mathrm{~g}$ unbalance. At the final location the trolleys make a $120^{\circ}$ angle while the theoretical expected value is $115^{\circ}$. This deviation is small and the balancing is efficient with a residual unbalance around $20 \mathrm{~g}$.

10 trajectories analysis issued from 10 consecutive runs are carried out for each tested configuration. The system is globally very efficient but a significant dispersion can be observed. The relative dispersion is higher when the initial imbalance to correct is low but in any case the residual imbalance is lesser than $100 \mathrm{~g}$ for $98 \%$ of the runs. The results for a $400 \mathrm{~g}$ unbalance at $1100 \mathrm{rpm}$ are plotted on Fig. 10. The system is very efficient and the residual unbalance is between $40 \mathrm{~g}$ and $100 \mathrm{~g}$ with this high initial unbalance. A $40 \mathrm{~g}$ residual unbalance is the maximum of achievable performance because the trolleys total mass is $360 \mathrm{~g}$.

Regardless the initial unbalance, the worst residual unbalance seems to be close to $100 \mathrm{~g}$. It could be due to

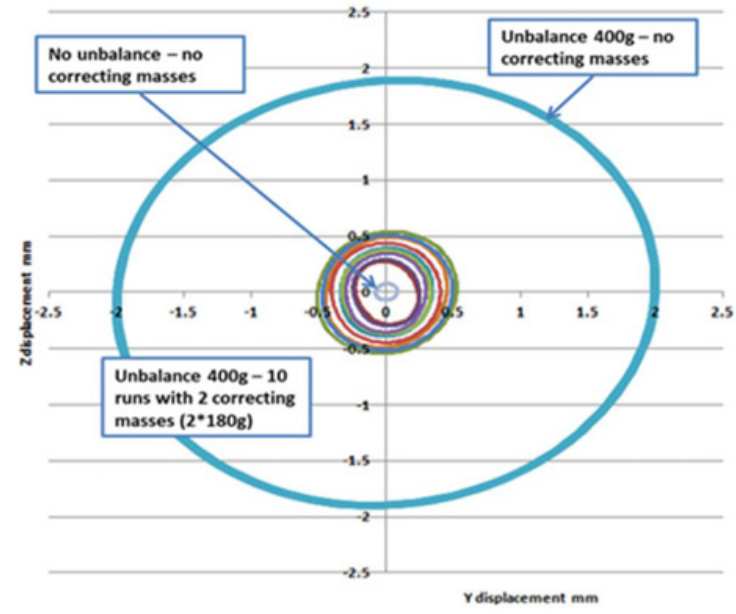

Figure 10. $1^{\text {st }}$ prototype. Drum displacements at $1100 \mathrm{rpm}$.

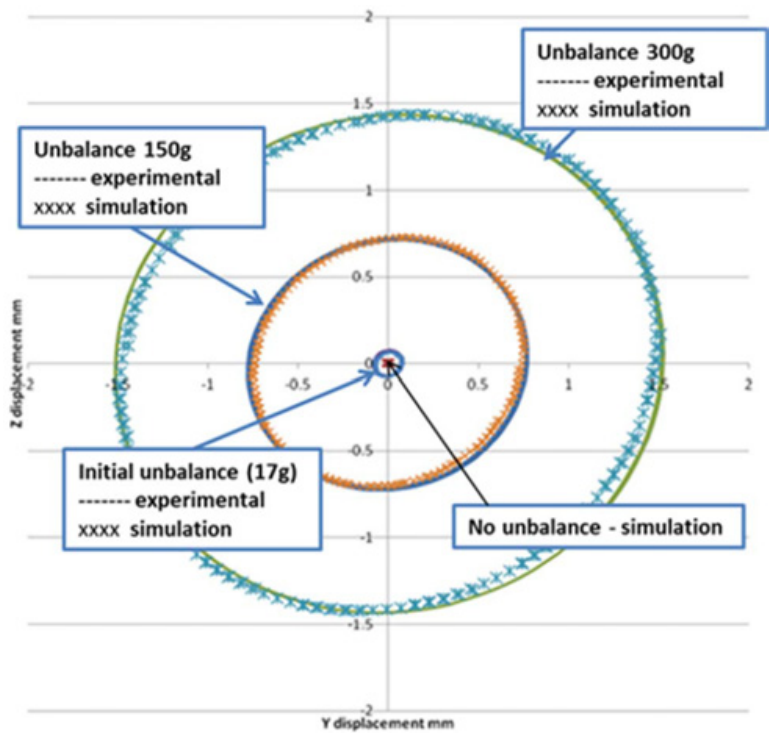

Figure 11. Simulation experimentation comparison at $1100 \mathrm{rpm}$.

a trolley wheel blocked on a rolling path junction: for the insertion in an existing drum, the ring is made of two half rings and there is a residual step at each ring junction. The optical observation of the trolleys locations confirms this assumption. The $100 \mathrm{~g}$ of residual unbalance could correspond to a threshold above of which the balancing forces are sufficient to free the trolley (to climb the step).

\subsection{Simulation vs. measure}

A computation measurement comparison is made in order to validate the model; the drum initial unbalance $(17 \mathrm{~g})$ is set in the model for improving the comparison. A good correlation is observable on Fig. 11.

\section{Measurements on the $2^{\text {nd }}$ prototype}

As a second prototype is required for final tests with water, the ring and trolleys design is modified for reducing the junction effects. Despite these modifications, steps remain at the rolling path junctions (probably due to the forces generated during the glue polymerisation inside the drum). 


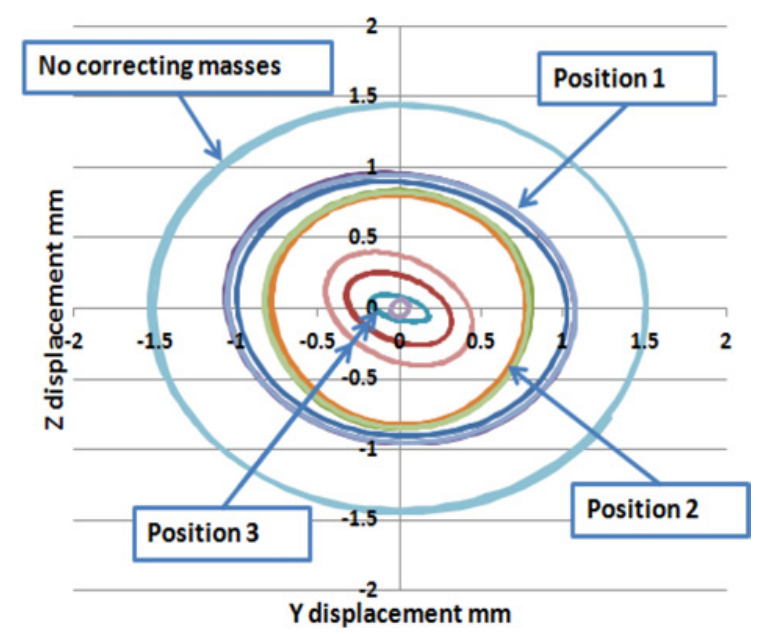

Figure 12. $2^{\text {nd }}$ prototype. Drum trajectories at $1100 \mathrm{rpm} .300 \mathrm{~g}$ unbalance at 3 different angular locations (3 runs for each of them).

Thus although the average performance is improved the maximum of residual unbalance remains around $100 \mathrm{~g}$. A sequence of tests is performed for observing the sensitivity to the unbalance location: the unbalance test mass is alternatively set on 3 different angular locations separated from $120^{\circ}$.

This test show a strong dependence from the initial unbalance location; this conclusion is enforced by the good reproducibility for a given unbalance location (see Fig. 12). These results seem to confirm that trolleys are blocked by geometrical defaults. A video observation is not possible for validating this assumption because this second prototype is equipped with a cover for waterproofness.

\section{Conclusions}

Designed with a multi-body dynamic model, an autobalancer has been mounted inside a washer-dryer drum. While other systems use generally balls for mobile masses, the LoWash system uses shaped trolleys allowing the ring cross section optimization and the control of the mobile masses behaviour at low speed. The test results highlight the efficiency of such a device but also the sensitivity to rolling track defects.
There are 2 ways to reduce the defects impact:

- Deleting or reducing the defect itself - it could be done by tooling the ring in one piece.

- Adding a soft rolling band on the wheel for facilitating the step crossing.

Because it can reduce significantly the drum vibrations and the dynamic loads on the drum bearing, the auto-balancer can be used for:

- Increasing the spin-drying speed.

- Lightening the hanging mass and components.

A work has to be done to adapt the system design to the requirements of washing machines massive production. The potential dissemination of the auto-balancer to other industrial machines (pumps, turbines, fans ...) has also to be investigated.

This work has been carried out within the framework of the LoWash project. LoWash is a project sustained by the "Research for SME" program of the FP7 which gather 4 SME (CDA, TEV, IDEA, and LINCIS), 1 white goods major company (VESTEL) and 2 RTD performers (HERI and VIBRATEC).

\section{References}

[1] A.H. Koevoets, "Automatic Balancing Unit an introduction", DCT 2001-27

[2] J. Adolfsson, "A study of stability in Autobalancing systems using multiple correction masses", Licentiate Thesis, Royal Institute of Technology (KTH), Stockholm, Sweden, 1997

[3] C. Lu, C. Hung, "Stability Analysis of a threeball automatic balancer", Journal of Vibration and Acoustics, Oct. 2008, 130

[4] N. Van de Wouw et al., "Performance of an automatic ball balancer with dry friction", International Journal of Bifurcation and Chaos, 15, $\mathrm{N}^{\circ} .1$ (2005) 65-82

[5] J.N. Bolton, "Single and dual-plane automatic balancing of an elastically mounted cylindrical rotor with considerations of coulomb friction and gravity", $\mathrm{PhD}$ Dissertation, 2010, Blacksburg, Virginia

[6] K. Olsson,, "Limits for the use of auto-balancing", International Journal of rotating Machinery, 10(3): 221-226, 2004 